\title{
Automotive Squint-forward-looking SAR: High Resolution and Early Warning
}

\author{
Ruizhi Hu, Member, IEEE, Bhavani Shankar Mysore Rama Rao, Senior Member, IEEE, \\ Ahmed Murtada, Student Member, IEEE, Mohammad Alaee-Kerahroodi, Member, IEEE, \\ and Björn Ottersten, Fellow, IEEE
}

\begin{abstract}
Forward-looking automotive radars can sense longdistant targets to enable early warning, but the lateral resolution is limited. Synthetic aperture radar (SAR) techniques can achieve very high azimuth resolution but cannot resolve targets in the forward direction. As a trade-off, squint-forward-looking SAR (SFL-SAR) can perform 2D imaging on a distant area squint to the moving direction, providing both high resolution and early warning. In this paper, we analyzed and derived the constraints of automotive SFL-SAR to satisfy both the required resolution and braking distance. Simulations and imaging results verified the analysis.
\end{abstract}

Index Terms-Automotive radar imaging, modified backprojection (BP), synthetic aperture radar (SAR), squint-forwardlooking.

\section{INTRODUCTION}

B ECAUSE of its unique all-weather all-day sensing ability, radar is an indispensable component for autonomous driving under harsh weather or light conditions [1]-[3]. In particular, millimeter-wave frequency-modulated continuouswave (FMCW) radar systems have been widely adopted in automotive sensing research and industry for the advantages of low cost, low power, small size, and easy signal preprocessings.

When considering the automobile's movement, it is natural to leverage the synthetic aperture radar (SAR) technique to obtain a high azimuth resolution. However, most automotive SAR works fall into the category of side-looking SAR, where the radar beam direction is near normal to the automotive motion. Although side-looking SAR can obtain high-resolution images, it is unable to detect the front-lying targets in advance. Consequently, its applications are limited to parking spot detection [4], [5], road boundary localization [6], infrastructure monitoring [7], and ground field sensing from elevated road [8].

Forward-looking is the most critical direction for safety concerns. Unfortunately, mono-static SAR fails in forwardlooking imaging because of its low lateral resolution and the left-right ambiguity problem [9]. As a result, the high and distance-invariant azimuth resolution of SAR can not be directly exploited in forward-looking radar imaging unless

This work was supported by the Luxembourg National Research Fund (FNR) through the CORE project "SPRINGER" under Grant C18/IS/12734677/SPRINGER. (Corresponding author: Ruizhi Hu.)

The authors are with the Interdisciplinary Centre for Security, Reliability and Trust (SnT), University of Luxembourg, L1885 Luxembourg City, Luxembourg (e-mail: ruizhi.hu@uni.lu; bhavani.shankar@uni.lu; ahmed.murtada@uni.lu; mohammad.alaee@uni.lu; bjorn.ottersten@uni.lu) advanced configurations like bi-static SAR are utilized [10][12]. Since bi-static SAR can change the spatial distribution of iso-range and iso-Doppler contours, particularly for the targets situated in the front, their range resolution and Doppler resolution vectors are no longer colinear so that $2 \mathrm{D}$ resolution can be obtained. However, an automobile's physical size cannot satisfy the requirement of a valid bi-static SAR configuration by itself, while using a signal from an airplane or satellite is too complicated and costly.

Hence for automotive radar imaging, real aperture radar array and multiple input multiple output (MIMO) radar arrays are extensively utilized to enhance lateral resolution in the forward direction [3], [13]. Unfortunately, the resulting resolution is still defined by angle and bounded by the array's physical constraints; hence the lateral resolution may be quite limited when targets are far from the radar. Consequently, the radar array cannot obtain azimuth resolution comparable to SAR. Although super-resolution beamforming techniques can be applied [14], [15], it will increase the computational overhead and only provide point cloud products [16]. Recently, some interesting techniques on enhancing the lateral resolution of forward-looking combining mechanical scanning with the SAR technique are proposed [17], [18], but the complexity of the imaging system increases.

As a compromise between forward-looking radar and sidelooking SAR, squint-forward-looking SAR (SFL-SAR) could be an excellent complement to existing radar imaging techniques. Due to the combined high lateral resolution and long forward sensing distance of SFL-SAR, it can provide reliable early warning for dangers originated from outside of the road or affecting all lanes. Some examples include fallen trees/buildings, landslides, tumbled rocks, a sunken road, a collapsed bridge, to name a few. The feasibility of automotive SFL-SAR has been demonstrated in [19], [20]. However, imaging performance and efficiency using frequency-domain algorithms at an extremely high squint angle and curved trajectory remain challenging [21], [22]. Regarding adapting BP to FMCW-SAR imaging, an additional image shift caused by radar's intra-pulse movement should be considered [23]. Under certain conditions such as large squint angle or high speed [24], modified BP should be utilized [7], [25].

In this paper, the constraints of SFL-SAR to satisfy both required $2 \mathrm{D}$ image resolution and safe distance for braking are derived, which can provide a reference for imaging area design. A longer synthetic aperture can enhance lateral resolution, but it will also leave a shorter distance for braking, 
causing a trade-off. Under certain constraints derived in this article, high lateral resolution and sufficient braking distance can be satisfied simultaneously for SFL-SAR. Finally, the resolution and localization accuracy of SFL-SAR imaging under an extremely high squint angle is verified via the modified BP algorithm. Specifically, the original contributions of our paper include:

1) System modelling of SFL-SAR for high resolution $2 \mathrm{D}$ imaging in the squint-forward direction;

2) Resolution analysis of SFL-SAR based on automotive parameters;

3) The novel concept of imaging area analysis to satisfy high resolution and sufficient braking distance simultaneously;

4) Corroboration the analysis via simulation results.

The remainder of this paper is organized as follows. In Section II, the signal model of FMCW-SAR is given. Section III presents the derivation of range and Doppler resolution vectors and the $2 \mathrm{D}$ resolution analysis in detail. In Section IV, constraints on 2D resolution and sufficient braking distance are derived for SFL-SAR, and imaging area design examples are shown. In Section V, the modified BP algorithm is introduced, and simulation imaging results are given. Finally, Section VII concludes the paper.

\section{Automotive FMCW-SAR Signal Model}

Let the FMCW radar system mounted on an automobile constantly transmit long pulses of the form,

$$
s_{t}(t)=\exp \left[-j\left(2 \pi f_{0} t+\pi K t^{2}\right)\right], \quad|t|<T_{p} / 2,
$$

where $T_{p}$ is the sweep time, $f_{0}$ is the carrier frequency, $K=B / T_{p}$ is the chirp rate, and $B$ is the bandwidth of the transmitted signal.

Based on the first-order Born approximation and the assumption of isotropic scattering [25], the received signal can be written as

$$
s_{r}(m, t)=\int_{\Omega} \sigma(\mathbf{s}) s_{t}(t-\tau[\mathbf{s}, \mathbf{r}(m, t)]) d \mathbf{s}
$$

where $m \in N$ is the index of pulses, $\Omega$ is the radar beam footprint, $\sigma(\mathbf{s})$ is the reflectivity at ground location $\mathbf{s}, \mathbf{r}(m, t)$ is the radar position at fast time $t$ during the $m$-th pulse, $\tau(\mathbf{s}, m, t)$ is the two-way delay from the radar to the target at $\mathbf{s}$ and then back to the radar.

For pulsed radar systems where the stop-go assumption is usually adopted, $\mathbf{r}$, and corresponding $\tau$, are assumed to vary with $m$ but not $t$ because of the short pulse duration. For an FMCW radar system, the stop-go assumption may fail, and the radar's intra-pulse movement with $t$ should be considered.

Let $r_{c}$ denote the reference range and $\tau_{c}=\frac{2 r_{c}}{c}$ denote the delay of the reference signal, where $c$ is the speed of light. After dechirp and range deskew [25], [26], the dechirped signal can be written as

$$
s_{d}(m, t)=\int_{\Omega} \sigma(\mathbf{s}) \exp j[\phi(\mathbf{s} ; m, t)] d \mathbf{s}
$$

where $\phi(\mathbf{s} ; m, t)$ is the phase that represents the ideal response of an isotropic scatter at $\mathbf{s}$, given by

$$
\phi(\mathbf{s} ; m, t)=2 \pi\left(f_{0}+K t-K \tau_{c}\right)\left[\tau(\mathbf{s}, m, t)-\tau_{c}\right]
$$

Let $R(\mathbf{s}, m, t)$ denote the instantaneous slant range from the target to the radar when transmitting and $R(\mathbf{s}, m, t+\tau)$ denote the instantaneous slant range when receiving. With regards to the automotive imaging scenario, the slant range is a few hundreds of meters at the most; hence the correponding $\tau$ is limited to a few microseconds. In comparison, $T_{p}$ for automotive FMCW radar is hundreds of microseconds, i.e., $\tau \ll T_{p}$, so the difference between $R(\mathbf{s}, m, t)$ and $R(\mathbf{s}, m, t+\tau)$ can be ignored in automotive radar imaging scenario, then the slant range can be assumed to vary with only $t$ and $m$, consequently,

$$
\begin{aligned}
\tau & =\tau(\mathbf{s}, m, t)=\frac{R(\mathbf{s}, m, t)+R(\mathbf{s}, m, t+\tau)}{c} \\
& \approx \frac{2 R(\mathbf{s}, m, t)}{c}=\frac{2\|\mathbf{s}-\mathbf{r}(m, t)\|}{c}
\end{aligned}
$$

\section{Resolution AnAlysis}

At the outset, we specialize the resolution properties of the automotive SAR imaging exploiting the peculiarities therein. For 2D-SAR imaging, the range resolution is provided by the wide bandwidth of the transmitted signal and is almost spatially-invariant except for the slant range to ground range projection. The cross-range resolution is achieved by the Doppler shift produced by the movement of the platform relative to the target. It is spatially-variant and is influenced by the velocity of the platform $\mathbf{v}$. In the following, inspired by [10] and based on the ground gradients of time delay and Doppler shift, the range resolution and Doppler resolution vectors for automotive SAR are derived. Building upon the two vectors, the $2 \mathrm{D}$ resolution can then be determined by their magnitudes and angle.

\section{A. Range Resolution Vector}

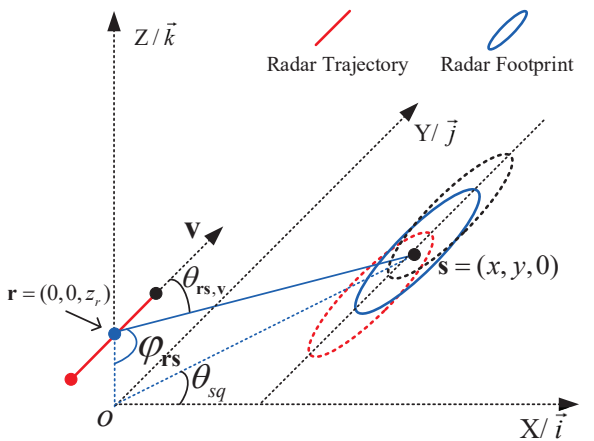

Fig. 1: Geometry of automotive FMCW-SAR.

To simplify analysis without loss of generality, the coordinates are defined with respect to the instantaneous radar location $\mathbf{r}$ and velocity $\mathbf{v}$, as shown in Fig. 1. The forward direction is along that of $\mathbf{v}$ and is denoted by the ground $\mathrm{Y}$ axis. The lateral direction is denoted by $X$ axis and the vertical direction is denoted by $\mathrm{Z}$ axis. Let $z_{r}$ denote the height of the radar; then the coordinate of $\mathbf{r}$ is $\left(0,0, z_{r}\right)$. Further, let an 
arbitrary ground target $\mathbf{s}$ have coordinates $(x, y, 0)$. Let $\vec{i}, \vec{j}$, and $\vec{k}$ denote the corresponding unit vectors of $\mathrm{X}, \mathrm{Y}$, and $\mathrm{Z}$. Let $\vec{u}_{\mathbf{r s}}$ denote the unit vector from $\mathbf{r}$ to $\mathbf{s}, \vec{u}_{\mathbf{r s}, g}$ denotes the unit vector along the ground projection of $\vec{u}_{\mathrm{rs}}$, and $\varphi_{\mathrm{rs}}$ denotes the instantaneous angle between $\vec{u}_{\text {rs }}$ and $-\vec{k}$, from Fig. 1, we have

$$
\begin{aligned}
& \vec{u}_{\mathbf{r s}}=\frac{1}{\|\mathbf{r}-\mathbf{s}\|}\left(x \vec{i}+y \vec{j}-z_{r} \vec{k}\right)=\frac{x \vec{i}+y \vec{j}-z_{r} \vec{k}}{\sqrt{x^{2}+y^{2}+z_{r}^{2}}} \\
& \vec{u}_{\mathbf{r s}, g}=\frac{1}{\|\mathbf{r}-\mathbf{s}\| \sin \varphi_{\mathbf{r s}}}(x \vec{i}+y \vec{j})=\frac{x \vec{i}+y \vec{j}}{\sqrt{x^{2}+y^{2}}} \\
& \sin \varphi_{\mathbf{r s}}=\frac{\sqrt{x^{2}+y^{2}}}{\sqrt{x^{2}+y^{2}+z_{r}^{2}}}
\end{aligned}
$$

Based on the delay in (5), a change in $\mathrm{s}$ will result in a change in $\tau$ via

$$
d \tau=\vec{d} \mathbf{s} \cdot \nabla_{g} \tau
$$

where $\nabla_{g} \tau$ is the ground gradient of $\tau$ that represents the direction of maximum change of the delay on the ground, and is given by

$$
\nabla_{g} \tau=\left[\frac{\partial \tau}{\partial x} \vec{i}+\frac{\partial \tau}{\partial y} \vec{j}\right]=\frac{2 \sin \varphi_{\mathbf{r s}}}{c} \vec{u}_{\mathbf{r s}, g}
$$

Since only a delay larger than $1 / B$ can be measured by the radar system, according to (7) and (8), the ground range resolution vector $\vec{\delta}_{r}(\mathbf{s})$ can be calculated using the unit norm property of $\vec{u}_{\mathbf{r s}, g}$

$$
\vec{\delta}_{r}(\mathbf{s})=\frac{1}{B\left\|\nabla_{g} \tau\right\|} \vec{u}_{\mathbf{r s}, g}=\frac{c}{2 B \sin \varphi_{\mathbf{r s}}} \vec{u}_{\mathbf{r s}, g}
$$

Different from air-borne SAR or space-borne SAR, the height of an automotive radar $z_{r}$ can be much smaller than the sensing distance. In (6), because of a small $z_{r}$, we have $\sin \varphi_{\mathbf{r s}} \rightarrow 1$. Further in (9), $\left\|\vec{\delta}_{r}(\mathbf{s})\right\| \rightarrow \frac{c}{2 B}$ is approximately spatially-invariant. However, the direction of $\vec{\delta}_{r}(\mathbf{s})$ is spatiallyvariant and is determined by $\vec{u}_{\mathbf{r s}, g}$.

\section{B. Doppler Resolution Vector}

The Doppler shift of the signal from $\mathbf{s}$ at radar position $\mathbf{r}$ is given by

$$
\begin{aligned}
d_{o} & =\frac{2 \mathbf{v} \cdot \vec{u}_{\mathbf{r s}}}{\lambda}=\frac{2\|\mathbf{v}\| \cos \theta_{\mathbf{r s}, \mathbf{v}}}{\lambda} \\
& =\frac{2\|\mathbf{v}\|}{\lambda} \frac{y}{\sqrt{x^{2}+y^{2}+z_{r}^{2}}}
\end{aligned}
$$

where $\lambda=c / f_{0}$ is the carrier wavelength and $\theta_{\mathbf{r s}, \mathbf{v}}$ is the angle between $\mathbf{v}$ and $\vec{u}_{\text {rs }}$.

The ground gradient of $d_{o}$ can be calculated via

$$
\begin{aligned}
\nabla_{g} d_{o} & =\left[\frac{\partial d_{o}}{\partial x} \vec{i}+\frac{\partial d_{o}}{\partial y} \vec{j}\right]=\frac{2\|\mathbf{v}\|}{\lambda} \nabla_{g}\left(\cos \theta_{\mathbf{r s}, \mathbf{v}}\right) \\
& =\frac{2\|\mathbf{v}\|}{\lambda\left(x^{2}+y^{2}+z_{r}^{2}\right)^{\frac{3}{2}}}\left[-x y \vec{i}+\left(x^{2}+z_{r}^{2}\right) \vec{j}\right]
\end{aligned}
$$

where $\nabla_{g}\left(\cos \theta_{\mathbf{r s}, \mathbf{v}}\right)$ is the ground gradient of $\cos \theta_{\mathbf{r s}, \mathbf{v}}$ and the modulus of $\nabla_{g} d_{o}$ is

$$
\begin{aligned}
\left\|\nabla_{g} d_{o}\right\| & =\frac{2\|\mathbf{v}\|}{\lambda}\left\|\nabla_{g}\left(\cos \theta_{\mathbf{r s}, \mathbf{v}}\right)\right\| \\
& =\frac{2\|\mathbf{v}\|\left[x^{2} y^{2}+\left(x^{2}+z_{r}^{2}\right)^{2}\right]^{\frac{1}{2}}}{\lambda\left(x^{2}+y^{2}+z_{r}^{2}\right)^{\frac{3}{2}}}
\end{aligned}
$$

Similar to the range resolution derivation, since the radar system can only measure a frequency difference larger than the reciprocal of synthetic aperture time $T_{s y n}$, the Doppler resolution vector can be calculated by

$$
\begin{aligned}
\vec{\delta}_{d_{o}}(\mathbf{s}) & =\frac{1}{T_{\text {syn }}\left\|\nabla_{g} d_{o}\right\|} \vec{u}_{d_{o}, g} \\
& =\frac{\lambda}{2 T_{\text {syn }}\|\mathbf{v}\|\left\|\nabla_{g}\left(\cos \theta_{\mathbf{r s}, \mathbf{v}}\right)\right\|} \vec{u}_{d_{o}, g}
\end{aligned}
$$

where $\vec{u}_{d_{o}, g}$ denotes the unit vector of the ground Doppler gradient, given by

$$
\vec{u}_{d_{o}, g}=\frac{-x y \vec{i}+\left(x^{2}+z_{r}^{2}\right) \vec{j}}{\sqrt{x^{2} y^{2}+\left(x^{2}+z_{r}^{2}\right)^{2}}}
$$

From (12) and (13), we have

$$
\begin{aligned}
& \lim _{z_{r} \rightarrow 0}\left\|\vec{\delta}_{d_{o}}(\mathbf{s})\right\| \\
& =\lim _{z_{r} \rightarrow 0} \frac{\lambda\left(x^{2}+y^{2}+z_{r}^{2}\right)^{\frac{3}{2}}}{2 T_{\text {syn }}\|\mathbf{v}\|\left[x^{2} y^{2}+\left(x^{2}+z_{r}^{2}\right)^{2}\right]^{\frac{1}{2}}} \\
& =\frac{\lambda\left(x^{2}+y^{2}\right)}{2 T_{\text {syn }}\|\mathbf{v}\||x|}
\end{aligned}
$$

From (14) and (15), for a small $z_{r},\left\|\vec{\delta}_{d o}(\mathbf{s})\right\|$ is spatiallyvariant, so is its unit vector $\vec{u}_{d_{o}, g}$.

\section{C. $2 D$ Resolution}

For a SAR system, the 2D resolution depends not only on the magnitudes of range and Doppler resolution vectors, but also their vectorial angle. In [10], a useful metric to measure the spatially-variant $2 \mathrm{D}$ resolution of SAR is given by the pixel size $\rho(\mathbf{s})$, defined as

$$
\rho(\mathbf{s})=\frac{\left\|\vec{\delta}_{r}(\mathbf{s})\right\|\left\|\vec{\delta}_{d_{o}}(\mathbf{s})\right\|}{\sin \theta_{r d}(\mathbf{s})}
$$

where $\theta_{r d}(\mathbf{s}) \in[0, \pi]$ denotes the vector angle between $\vec{\delta}_{r}(\mathbf{s})$ and $\vec{\delta}_{d_{o}}(\mathbf{s})$, from (6) and (14),

$$
\begin{aligned}
\cos \theta_{r d}(\mathbf{s}) & =\frac{\vec{\delta}_{r}(\mathbf{s}) \cdot \vec{\delta}_{d_{o}}(\mathbf{s})}{\left\|\vec{\delta}_{r}(\mathbf{s})\right\|\left\|\vec{\delta}_{d_{o}}(\mathbf{s})\right\|}=\vec{u}_{\mathbf{r s}, g} \cdot \vec{u}_{d_{o}, g} \\
& =\frac{y z_{r}^{2}}{\sqrt{\left[x^{2} y^{2}+\left(x^{2}+z_{r}^{2}\right)^{2}\right]\left(x^{2}+y^{2}\right)}}
\end{aligned}
$$

Naturally, smaller $\rho(\mathbf{s})$ implies higher resolution. Specifically, when $x \rightarrow 0, \lim _{x \rightarrow 0} \cos \theta_{r d}(\mathbf{s})=\frac{y z_{r}^{2}}{\sqrt{z_{r}^{4} y^{2}}}= \pm 1$, which 
means $\sin \theta_{r d}(\mathbf{s}) \rightarrow 0$. Further from (16), we have $\rho(\mathbf{s}) \rightarrow \infty$. Hence, for a target directly in the front or rear of the vehicle, $\vec{\delta}_{r}(\mathbf{s})$ and $\vec{\delta}_{d_{o}}(\mathbf{s})$ are colinear and $2 \mathrm{D}$ resolution is not feasible, which explains why SAR is not an option for forward direction detection. On the contrary, for side-looking where $y \rightarrow 0$, we have $\lim _{y \rightarrow 0} \cos \theta_{r d}(\mathbf{s})=0$, which means $\vec{\delta}_{r}(\mathbf{s})$ and $\vec{\delta}_{d_{o}}(\mathbf{s})$ are orthogonal, thus SAR can provide the finest $2 \mathrm{D}$ resolution.

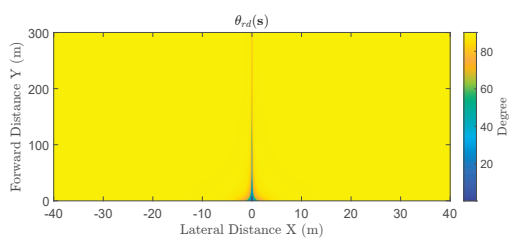

(a)

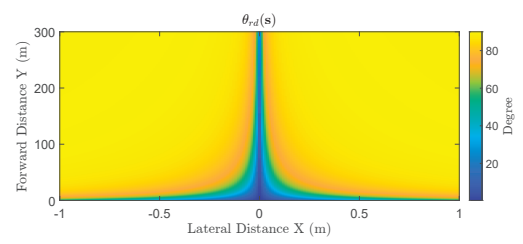

(b)

Fig. 2: Spatial variation of $\theta_{r d}(\mathbf{s})$. (a) Entire lateral and forward range. (b) Focus on lateral range.

Due to the small $z_{r}$ in automotive radar, based on (17), when $|x|$ or $|y|$ is large enough, it also has $\cos \theta_{r d}(\mathbf{s}) \rightarrow 0$. For example, when $z_{r}=1.5 \mathrm{~m}$, the value of $\theta_{r d}(\mathbf{s})$ for a lateral range of $x \in[-40 \mathrm{~m}, 40 \mathrm{~m}]$ and a forward range of $y \in[0 \mathrm{~m}, 300 \mathrm{~m}]$ is shown in Fig. 2(a), with Fig. 2(b) depicting the horizontal zoom-in image. Because of the particular small $z_{r}$ in automotive SAR, in Fig. 2, for most of the surrounding area that is deviated from the forward direction, $\theta_{r d}(\mathbf{s}) \approx 90^{\circ}$ is satisfied as predicted. For these areas, since both $\left\|\vec{\delta}_{r}(\mathbf{s})\right\|$ and $\sin \theta_{r d}(\mathbf{s})$ in (16) are approximately spatially-invariant, the spatially-variant part of $\rho(\mathbf{s})$ for automotive SAR is predominantly governed by $\left\|\vec{\delta}_{d_{o}}(\mathbf{s})\right\|$.

TABLE I: Simulation parameters and values

\begin{tabular}{|c|c|c|c|c|c|}
\hline$f_{0}$ & $B$ & $\rho_{0}$ & $z_{r}$ & $\|\mathbf{v}\|$ & $L_{\text {syn }}$ \\
\hline $77 \mathrm{GHz}$ & $1.5 \mathrm{GHz}$ & $0.01 \mathrm{~m}^{2}$ & $1.5 \mathrm{~m}$ & $32 \mathrm{~km} / \mathrm{h}$ & $30 \mathrm{~m}$ \\
\hline
\end{tabular}

Let $\rho_{0}$ denote a desired standard $2 \mathrm{D}$ resolution and $\rho(\mathbf{s})=$ $\rho_{0} N_{\rho}(\mathbf{s})$, where $N_{\rho}(\mathbf{s})$ is the corresponding spatial variation normalized to $\rho_{0}$. A larger $N_{\rho}(\mathbf{s})$ indicates a lower $2 \mathrm{D}$ resolution because a point target located there will have a larger main-lobe area. Based on the parameters listed in Table. I, the contours of $N_{\rho}(\mathbf{s})$ over the same area of Fig. 2 is shown in Fig. 3. According to the contours, 2D resolution gets lower with an increasing $y$ or decreasing $|x|$. This is undesirable since we want to cover a large imaging area that could be distant and close to the front at the same time, so a trade-off should be made between $y$ and $|x|$.

Besides the resolution failure in the forward direction, mono-static forward-looking SAR also has the left-right ambiguity because two targets that are symmetric about $\mathrm{Y}$ axis have the same range and Doppler history. Consequently, if we want

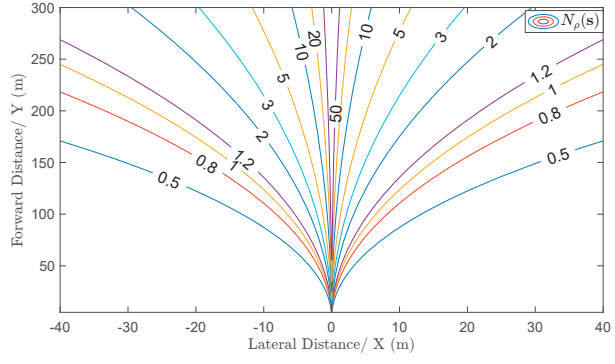

Fig. 3: Contours of the 2D resolution as a function of lateral and forward range provided.

to image both sides, two radars separately illuminating each side are needed. Because the $2 \mathrm{D}$ resolution is symmetric about $\mathrm{Y}$ axis shown in Fig. 3, in the remainder of this paper, we only consider the case of right side SFL-SAR for simplicity. The results for the left side are similar.

\section{IMAGING AREA ANALYSIS FOR SFL-SAR}

In the last section, we have derived the $2 \mathrm{D}$ resolution for mono-static automotive SAR under linear trajectory with constant velocity, and we also have analyzed the reason for its failure on forward-looking imaging. On that basis, in this section, we will further discuss the constraints of SFL-SAR on the imaging area to achieve both the desired resolution and sufficient stopping distance in case of detected obstacle.

Combining (9), (13), and (16), for an arbitrary target s, we have

$$
\rho(\mathbf{s}) \propto \frac{\lambda}{B\|\mathbf{v}\| T_{\text {syn }}}=\frac{\lambda}{B L_{\text {syn }}}
$$

where $L_{\text {syn }}=\|\mathbf{v}\| T_{\text {syn }}$ is the synthetic aperture length given a linear constant velocity.

As for millimeter-wave automotive radar, $\lambda$ is relatively small and $B$ is relatively large, both providing advantages in obtaining high resolution. Additionally, for different values of $\|\mathbf{v}\|$, a stable resolution can be obtained by setting a fixed $L_{\text {syn }}$ by adapting $T_{s y n}$. This invariance will be beneficial for the target detection via both human eyes and machine algorithms.

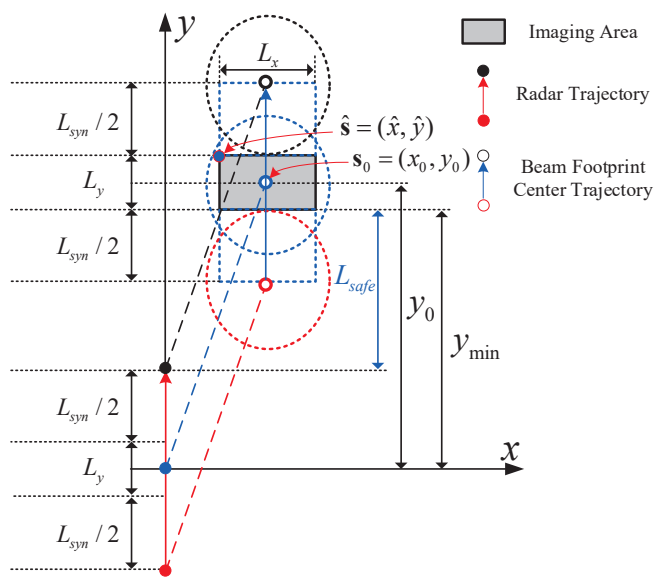

Fig. 4: Top view of automotive SFL-SAR imaging.

Fig. 4 shows the top view of the scenario of Fig. 1, where $\mathbf{s}_{\mathbf{0}}=\left(x_{0}, y_{0}\right)$ denotes the center of an rectangular imaging 
area, and $L_{x}$ and $L_{y}$ are the extents along lateral and forward direction, respectively. Because of the $2 \mathrm{D}$ resolution variation pattern shown in Fig. 3, the poorest $2 \mathrm{D}$ resolution within the imaging area will occur on the left edge and at its farthest distance from the radar. Let $\hat{\mathbf{s}}=(\hat{x}, \hat{y})=\left(x_{0}-L_{x} / 2, y_{0}+\right.$ $\left.L_{y} / 2\right)$ denote the upper left corner of the area. As illustrated in Fig. 4, to achieve the required 2D resolution for the whole imaging area, the radar should travel in a linear trajectory with a length of $L_{y}+L_{\text {syn }}$. Let $\tilde{\mathbf{s}}=(\hat{x}, \tilde{y})$ be the location on the left edge that corresponds to the worst resolution, where $\tilde{y}$ denote the possible farthest distance from any target within the imaging area to the radar along $\mathrm{Y}$ axis. When the center of the radar trajectory is defined as the origin of the coordinates, we have

$$
\tilde{y}=\hat{y}+\left(L_{\text {syn }}-L_{y}\right) / 2=y_{0}+L_{\text {syn }} / 2
$$

Depending on the relative size of $L_{s y n}$ and $L_{y}, \tilde{y}$ could be larger or smaller than $\hat{y}$. Finally, the constraint on resolution can be defined as

$$
\rho(\tilde{\mathbf{s}})=\rho(\hat{x}, \tilde{y}) \leqslant \rho_{0}
$$

As illustrated in Fig. 4, $L_{s y n}$ and $L_{y}$ are limited when $\|\mathbf{v}\|$ is large, or the distance between the radar and the scene may be too close to stop the vehicle. According to the UK highway code [27], the stopping distance $L_{\text {stop }}$ is the sum of the thinking distance $L_{\text {think }}$ and the braking distance $L_{\text {brake }}$, and the typical values are listed in Table. II.

TABLE II: Stopping distances under different velocity

\begin{tabular}{|c|c|c|c|c|c|c|}
\hline$\|\mathbf{v}\|(\mathrm{km} / \mathrm{h})$ & 32 & 48 & 64 & 80 & 96 & 112 \\
\hline$L_{\text {think }}(\mathrm{m})$ & 6 & 9 & 12 & 15 & 18 & 21 \\
\hline$L_{\text {brake }}(\mathrm{m})$ & 6 & 14 & 24 & 38 & 55 & 75 \\
\hline$L_{\text {stop }}(\mathrm{m})$ & 12 & 23 & 36 & 53 & 73 & 96 \\
\hline
\end{tabular}

In terms of autonomous driving, $L_{\text {think }}$ of a human driver can be possibly replaced by $L_{s p}$, a much shorter time for learning and processing algorithms. Then, $L_{\text {stop }}$ can be set as

$$
L_{\text {stop }}=(1+\gamma) L_{\text {brake }}
$$

where $\gamma>0$ is a factor considering $L_{s p}$ and certain marginal safe distance.

Let $L_{\text {safe }}$ denote the minimum distance between the radar and the closest target in the imaging area at the end of radar trajectory along Y axis, as illustrated in Fig. 4, we have

$$
L_{\text {safe }}=y_{0}-L_{y}-L_{\text {syn }} / 2=\tilde{y}-L_{\text {syn }}-L_{y}
$$

Let $L_{\text {safe }} \geq L_{\text {stop }}$, then the constraint on safety distance for the imaging area is given by

$$
L_{y} \leq \tilde{y}-L_{\text {syn }}-L_{\text {stop }}
$$

With $L_{y}$ determined, the minimum $y$ coordinate of the imaging area is

$$
y_{\min }=\hat{y}-L_{y}=\tilde{y}-L_{s y n} / 2-L_{y} / 2
$$

Let $\hat{x}=15 \mathrm{~m}, L_{x}=20 \mathrm{~m}, \gamma=0.1$. Further, for the parameters in Table. I, imaging area constraints for SFL-SAR imaging are derived in Table. III and shown in Fig. 5 for different values of $\|\mathbf{v}\|, L_{\text {syn }}$ and the corresponding $L_{\text {brake }}$

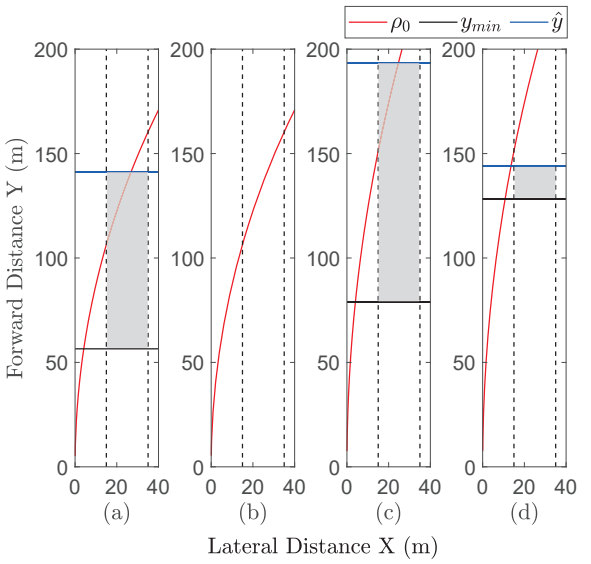

Fig. 5: Illustration of the imaging area constraints with shaded region depicting feasible regions. (a) $L_{\text {syn }}=15 \mathrm{~m},\|\mathbf{v}\|=$ $32 \mathrm{~km} / \mathrm{h}$. (b) $L_{\text {syn }}=15 \mathrm{~m},\|\mathbf{v}\|=112 \mathrm{~km} / \mathrm{h}$. (c) $L_{\text {syn }}=$ $30 \mathrm{~m},\|\mathbf{v}\|=32 \mathrm{~km} / \mathrm{h}$. (d) $L_{\text {syn }}=30 \mathrm{~m},\|\mathbf{v}\|=112 \mathrm{~km} / \mathrm{h}$.

from Table. II. In Fig. 5, the red lines denote the contours of $\rho_{0}$ calculated by (16). Then $\tilde{y}$ is determined by the intersection points of the red and dotted lines of $\hat{x}=15 \mathrm{~m}$, and subsequently $L_{y}$ can be determined by (23). After that, $\hat{y}$ (blue lines) can be determined according to (19) and $y_{\min }$ (black lines) can be determined according to (24). It is shown that the contours of $\rho_{0}$ are determined by $L_{s y n}$ rather than $\|\mathbf{v}\|$. The area satisfying both the resolution constraint and the safety distance constraint is shaded by grey in Fig. 5, and the corresponding values of $\tilde{y}, L_{y}, L_{\text {stop }}, \hat{y}$, and $y_{\text {min }}$ are listed in Table. III. Specifically, in Fig. 5(b), the two constraints can not be satisfied simultaneously because that will lead to $L_{y}<0$.

TABLE III: Imaging area constraints

\begin{tabular}{|c|c|c|c|c|c|}
\hline$L_{\text {syn }} \&\|\mathbf{v}\|$ & $\tilde{y}$ & $L_{y}$ & $L_{\text {stop }}$ & $\hat{y}$ & $y_{\min }$ \\
\hline $15 \mathrm{~m}, 32 \mathrm{~km} / \mathrm{h}$ & $106.4 \mathrm{~m}$ & $84.8 \mathrm{~m}$ & $6.6 \mathrm{~m}$ & $141.3 \mathrm{~m}$ & $56.5 \mathrm{~m}$ \\
\hline $15 \mathrm{~m}, 112 \mathrm{~km} / \mathrm{h}$ & $106.4 \mathrm{~m}$ & $/$ & $105.6 \mathrm{~m}$ & $/$ & $/$ \\
\hline $30 \mathrm{~m}, 32 \mathrm{~km} / \mathrm{h}$ & $151.2 \mathrm{~m}$ & $114.6 \mathrm{~m}$ & $6.6 \mathrm{~m}$ & $193.5 \mathrm{~m}$ & $78.9 \mathrm{~m}$ \\
\hline $30 \mathrm{~m}, 112 \mathrm{~km} / \mathrm{h}$ & $151.2 \mathrm{~m}$ & $15.6 \mathrm{~m}$ & $105.6 \mathrm{~m}$ & $144 \mathrm{~m}$ & $128.4 \mathrm{~m}$ \\
\hline
\end{tabular}

\section{Modified BACK-PROJECTION IMAGING}

To cover an effective imaging area for safety, a large $y$ and a small $x$ are desired, which means the squint angle can be extremely large for SFL-SAR. For example in Fig. 5(d), the squint angle between $\hat{\mathbf{s}}(\hat{x}, \hat{y})$ and the radar at the trajectory center is $\theta_{s q}(\hat{\mathbf{s}})=\arctan \left(\frac{144}{15}\right)=84.05^{\circ}$. Current frequencydomain imaging algorithms fail in air-borne and space-borne SAR scenarios for such a large squint angle because of the severe coupling between range and Doppler gradients. Fortunately, as shown in Fig. 2, because of the small $z_{r}$ of automotive SAR, the range and Doppler resolution vectors stay orthogonal for most of the imaging area. This may lead to effective processing in the frequency domain, which is beyond the scope of this paper.

In this paper, we utilize the modified BP algorithm of FMCW-SAR [25] to verify the feasibility of high-squint imaging and the correctness of our resolution analysis. Exploiting 
the stop-go assumption, conventional $\mathrm{BP}$ is equivalent to a zero-order approximation of $\tau(\mathbf{s}, m, t)$ in (5) given by $\tau_{0 m}$

$$
\tau_{0 m}=\frac{2\|\mathbf{s}-\mathbf{r}(m, t=0)\|}{c}
$$

When the intra-pulse movement of FMCW-SAR [25], [26] can not be ignored, a better approximation of $\tau(\mathbf{s}, m, t)$ is to use the first-order approximation as,

$$
\tau(\mathbf{s}, m, t) \approx \tau_{0 m}+\tau_{1 m} t
$$

where $\tau_{1 m}$ is given by

$$
\begin{aligned}
& \tau_{1 m}=\left.\frac{\partial \tau(\mathbf{s}, m, t)}{\partial t}\right|_{t=0}=\frac{\mathbf{v} \cdot(\mathbf{s}-\mathbf{r}(m, t=0))}{\|\mathbf{s}-\mathbf{r}(m, t=0)\|} \\
& =\mathbf{v} \cdot \vec{u}_{\mathbf{r s}}=\|\mathbf{v}\| \cos \theta_{\mathbf{r s}, \mathbf{v}} \\
& =\|\mathbf{v}\| \sin \theta_{s q} \sin \varphi_{\mathbf{s r}} \approx\|\mathbf{v}\| \sin \theta_{s q}
\end{aligned}
$$

Therefore, $\tau_{1 m}$ may not be ignored for high squint angle scenario because it is approximately proportional to $\sin \theta_{s q}$. Then, $\phi(\mathbf{s} ; m, t)$ in (4) can be rearranged as

$$
\phi(\mathbf{s} ; m, t)=2 \pi\left[\begin{array}{l}
\left(f_{0}+K t-K \tau_{c}\right) \tau_{c}-f_{0} \tau(\mathbf{s}, m, t)+\ldots \\
-K\left(t-\tau_{c}\right) \tau(\mathbf{s}, m, t)
\end{array}\right]
$$

As $f_{0} \gg\left|K\left(t-\tau_{c}\right)\right|$, we can use first-order approximation of $\tau(\mathbf{s}, m, t)$ for the second term in (28) and the zeroorder approximation for the third term [25], to approximate $\phi(\mathbf{s} ; m, t)$ as

$$
\begin{aligned}
& \hat{\phi}(\mathbf{s} ; m, t) \\
& =2 \pi\left[\begin{array}{l}
\left(f_{0}+K t-K \tau_{c}\right) \tau_{c}-f_{0}\left(\tau_{0 m}+\tau_{1 m} t\right)+\ldots \\
-K\left(t-\tau_{c}\right) \tau_{0 m}
\end{array}\right] \\
& =2 \pi\left(A_{m} t+B_{m}\right)
\end{aligned}
$$

where $A_{m}$ and $B_{m}$ are defined as

$$
\begin{aligned}
& A_{m}=K \tau_{c}-K \tau_{0 m}-f_{0} \tau_{1 m} \\
& B_{m}=\left(f_{0}-K \tau_{c}\right)\left(\tau_{c}-\tau_{0 m}\right)
\end{aligned}
$$

Through the approximation, the modified BP image is thus obtained by an approximated 2D matched-filtering of $s_{d}(m, t)$ via

$$
\begin{aligned}
& \hat{\sigma}(\mathbf{s})=\sum_{m} \int s_{d}(m, t) \exp [-j \hat{\phi}(\mathbf{s} ; m, t)] d t \\
& =\sum_{m} \exp \left(-j 2 \pi B_{m}\right) \int s_{d}(m, t) \exp \left[-j 2 \pi A_{m} t\right] d t \\
& =\sum_{m} \exp \left(-j 2 \pi B_{m}\right) S\left(A_{m}\right)
\end{aligned}
$$

where $S$ is the Fourier transform with respect to $t, A_{m}$ is the energy curve of target $\mathbf{s}$ with respect to $m$ after range compression.

Compared with conventional BP, the extra term of $f_{0} \tau_{1 m}$ in $A_{m}$ will cause an extra shift $\Delta R_{m}$ along range direction in the range-compressed curve, which is

$$
\begin{aligned}
\Delta R_{m} & =\frac{c f_{0} \tau_{1 m}}{2 K}=\frac{c f_{0} T_{p}\|\mathbf{v}\| \cos \theta_{\mathbf{r s}, \mathbf{v}}}{2 B} \\
& \approx \frac{c f_{0} T_{p}\|\mathbf{v}\| \sin \theta_{s q}}{2 B}
\end{aligned}
$$

For automotive FMCW-SAR, $f_{0}$ and $T_{p}$ are relatively large. Additionally, for large squint angles and large velocities, $\Delta R_{m}$ may be too large to ignore, thereby motivating the use of modified BP instead of conventional BP.

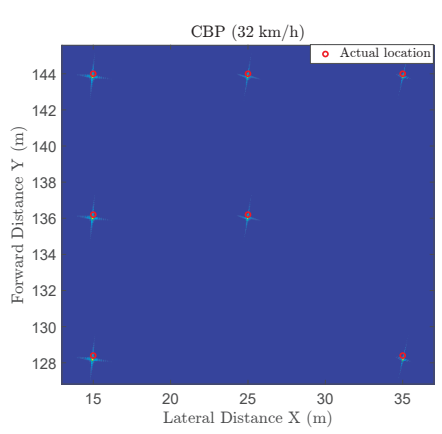

(a)

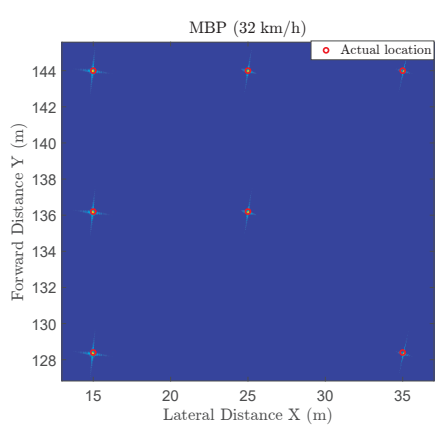

(c)

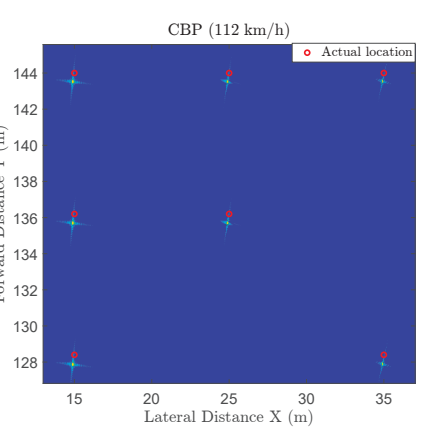

(b)

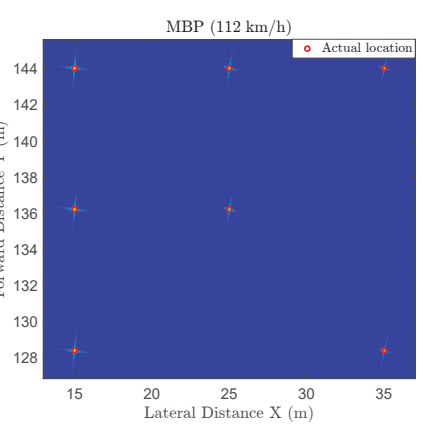

(d)
Fig. 6: (a) Conventional BP $(\|\mathbf{v}\|=32 \mathrm{~km} / \mathrm{h})$; (b) Conventional BP $(\|\mathbf{v}\|=112 \mathrm{~km} / \mathrm{h})$; (c) Modified BP $(\|\mathbf{v}\|=$ $32 \mathrm{~km} / \mathrm{h})$; (d) Modified BP $(\|\mathbf{v}\|=112 \mathrm{~km} / \mathrm{h})$.

\section{RESUlTs AND Discussion}

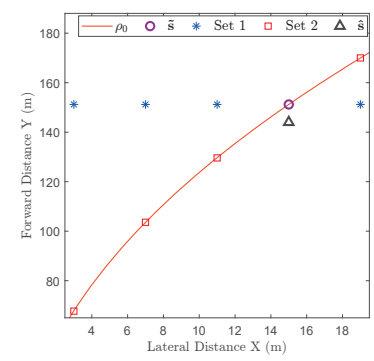

Fig. 7: Simulation setup for resolution comparison.

For the imaging area given in Fig. 5(d), we set $T_{p}=300 \mu \mathrm{s}$ and PRI $=350 \mu \mathrm{s}$, and use two different velocities $\|\mathbf{v}\|=$ $32 \mathrm{~km} / \mathrm{h}$ and $\|\mathbf{v}\|=112 \mathrm{~km} / \mathrm{h}$ with other parameters in Table. I in the simulations. The imaging results are shown in Fig. 6, where the actual location of targets are denoted by the red circles. In each figure, all targets are well focused, with only slightly different resolution and side-lobe direction because of the spatial variation of resolution vectors. In Fig. 6(a) and (b), targets' reconstructed location are deviated from their actual location, and the offset in Fig. 6(b) is larger than Fig. 6(a) 


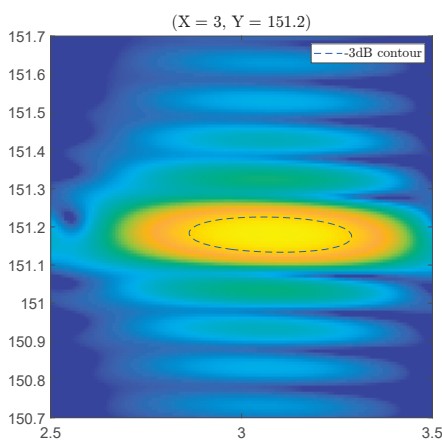

(a)

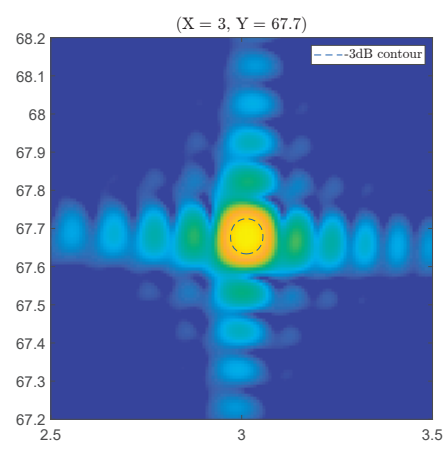

(e)

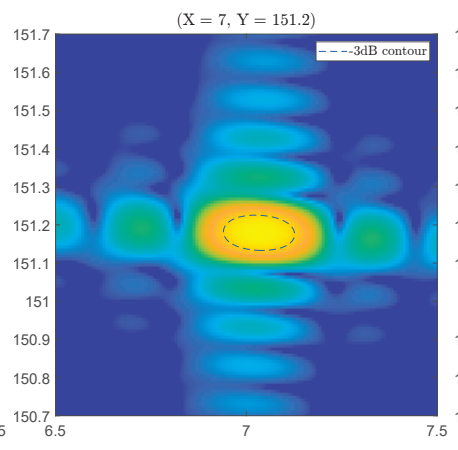

(b)

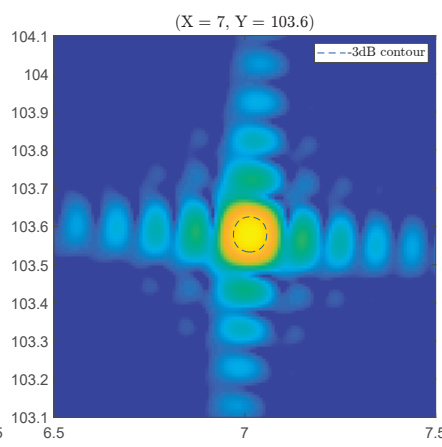

(f)

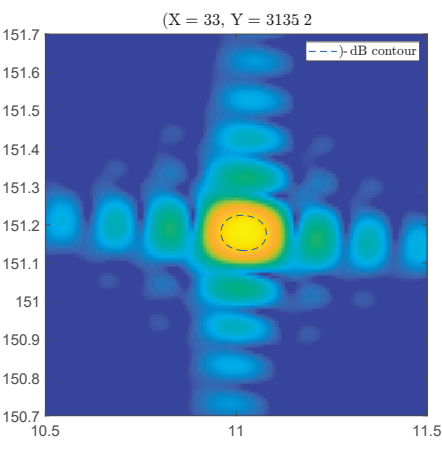

(c)

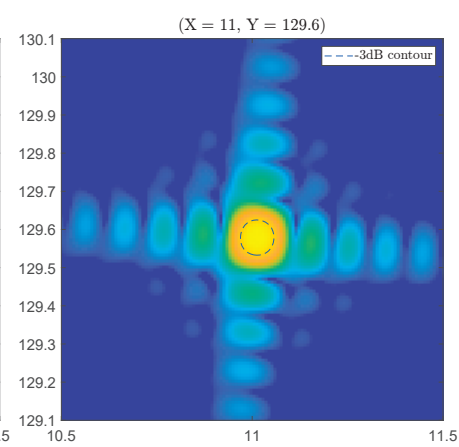

(g)

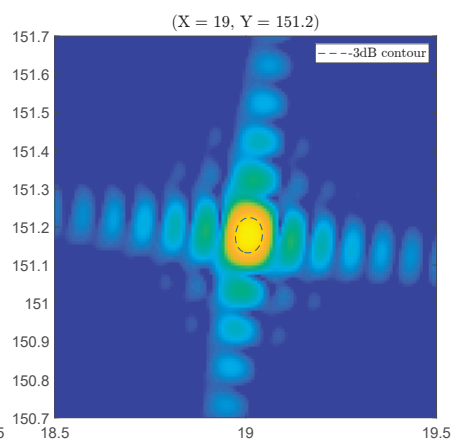

(d)

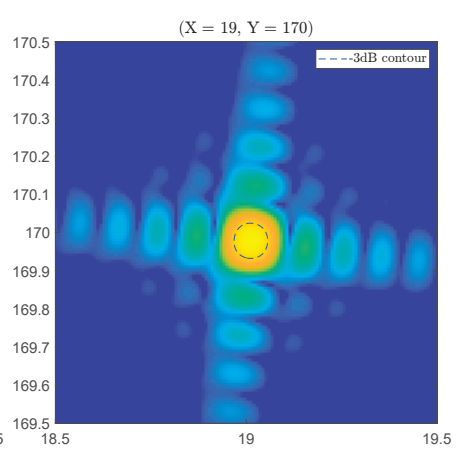

(h)

Fig. 8: Imaging results at the two sets. (a-d) Set 1; (e-f) Set 2.

because of the larger velocity. As a comparison, in Fig. 6(c) and (d), all targets are focused on their actual location.

To better verify our analysis on the spatial resolution variation, we further present zoomed images around several point targets using the modified BP algorithm [25]. Here, we set $L_{\text {syn }}=30 \mathrm{~m},\|\mathbf{v}\|=112 \mathrm{~km} / \mathrm{h}$. The distribution of the targets is shown in Fig. 7, the circle represents $\tilde{\mathbf{s}}$, the triangle represents $\hat{\mathbf{s}}$, the red line represents the contour of $\rho_{0}$, the blue asterisks (Set 1) represent a set of targets with the same forward distance as $\tilde{\mathbf{s}}$ but with different lateral locations, the red squares (Set 2) represent the corresponding targets with the same lateral locations as Set 1 along the contour of $\rho_{0}$.

Fig. 9 depicts the results at $\tilde{\mathbf{s}}$ and $\hat{\mathbf{s}}$. The $-3 \mathrm{~dB}$ contours demonstrated that the resolution constraint of $\left(\rho(\tilde{\mathbf{s}}) \leqslant 0.01 \mathrm{~m}^{2}\right)$ are satisfied both for $\tilde{\mathbf{s}}$ and $\hat{\mathbf{s}}$. The lateral resolution at $\hat{\mathbf{s}}$ is slightly better than at $\tilde{\mathbf{s}}$ because of a slightly smaller forward distance. The range and Doppler side-lobe directions in both figures are orthogonal as predicted and their reconstructed locations are very accurate.

Fig. 8 are the results for targets at the two sets of targets in Fig. 7 with their location given in the title of each figure. As predicted by our analysis, the first row shows the lateral resolution deteriorates with an decrease in the lateral distance. Whereas in the second row, targets located on the same resolution contour demonstrate the equivalent resolution after imaging. Furthermore, by comparing each vertical pair of images in Fig. 8, it also validated that the lateral resolution worsens with an increase in the forward distance.

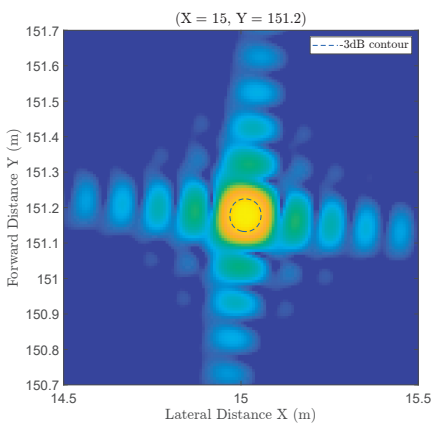

(a)

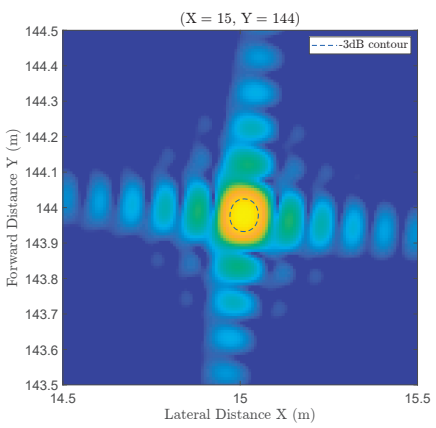

(b)
Fig. 9: Imaging results at (a) $\tilde{\mathbf{s}}$; (b) $\hat{\mathbf{s}}$.

\section{Conclusion}

While forward SAR techniques are known to offer little advantages in automotive, this paper explores the SAR application in the squint forward direction to enhance imaging resolution. In this paper, an analysis of the $2 \mathrm{D}$ resolution of automotive SAR is undertaken. The resulting expressions show that the key spatially-variant component of $2 \mathrm{D}$ resolution for automotive SAR is determined by the Doppler resolution vector. Based on the analysis, the constraints for SFL-SAR to satisfy both $2 \mathrm{D}$ resolution requirement and safety requirement are derived. Besides, the effectiveness of SFL-SAR under extremely high squint angle is verified by simulation via modified BP imaging algorithm, which demonstrated that SFLSAR is a viable technique in automotive radar for early ob- 
stacle detection in the squint forward direction. The next steps include efficient imaging algorithm and motion compensation research, real data collection and processing.

\section{REFERENCES}

[1] K. Bengler, K. Dietmayer, B. Farber, M. Maurer, C. Stiller, and H. Winner, "Three decades of driver assistance systems: Review and future perspectives," IEEE Intelligent transportation systems magazine, vol. 6, no. 4, pp. 6-22, 2014.

[2] I. Bilik, O. Longman, S. Villeval, and J. Tabrikian, "The rise of radar for autonomous vehicles: Signal processing solutions and future research directions," IEEE Signal Processing Magazine, vol. 36, no. 5, pp. 20-31, 2019.

[3] S. M. Patole, M. Torlak, D. Wang, and M. Ali, "Automotive radars: A review of signal processing techniques," IEEE Signal Processing Magazine, vol. 34, no. 2, pp. 22-35, 2017.

[4] H. Wu and T. Zwick, "Automotive sar for parking lot detection," in 2009 German microwave conference. IEEE, 2009, pp. 1-8.

[5] T. Kan et al., "Implementation of real-time automotive sar imaging," in 2020 IEEE 11th Sensor Array and Multichannel Signal Processing Workshop (SAM). IEEE, 2020, pp. 1-4.

[6] D. Clarke, D. Andre, and F. Zhang, "Synthetic aperture radar for lane boundary detection in driver assistance systems," in 2016 IEEE International Conference on Multisensor Fusion and Integration for Intelligent Systems (MFI). IEEE, 2016, pp. 238-243.

[7] S. Palm, R. Sommer, and U. Stilla, "Mobile radar mapping-subcentimeter sar imaging of roads," IEEE Transactions on Geoscience and Remote Sensing, vol. 56, no. 11, pp. 6734-6746, 2018.

[8] D.-H. Jung, H.-S. Kang, C.-K. Kim, J. Park, and S.-O. Park, "Sparse scene recovery for high-resolution automobile fmcw sar via scaled compressed sensing," IEEE Transactions on Geoscience and Remote Sensing, vol. 57, no. 12, pp. $10136-10146,2019$.

[9] S. Dai and W. Wiesbeck, "High resolution imaging for forward looking sar with multiple receiving antennas," in IGARSS 2000. IEEE 2000 International Geoscience and Remote Sensing Symposium. Taking the Pulse of the Planet: The Role of Remote Sensing in Managing the Environment. Proceedings (Cat. No. 00CH37120), vol. 5. IEEE, 2000, pp. 2254-2256.

[10] G. Cardillo, "On the use of the gradient to determine bistatic sar resolution," in Antennas and Propagation Society International Symposium, vol. 2, 1990, pp. 1032-1035.

[11] X. Qiu, D. Hu, and C. Ding, "Some reflections on bistatic sar of forwardlooking configuration," IEEE Geoscience and Remote Sensing Letters, vol. 5, no. 4, pp. 735-739, 2008.

[12] J. Wu, J. Yang, Y. Huang, H. Yang, and H. Wang, "Bistatic forwardlooking sar: Theory and challenges," in 2009 IEEE Radar Conference. IEEE, 2009, pp. 1-4.

[13] G. Hakobyan and B. Yang, "High-performance automotive radar: A review of signal processing algorithms and modulation schemes," IEEE Signal Processing Magazine, vol. 36, no. 5, pp. 32-44, 2019.

[14] Y. Guercan and A. Yarovoy, "Super-resolution algorithm for joint rangeazimuth-doppler estimation in automotive radars," in 2017 European Radar Conference (EURAD). IEEE, 2017, pp. 73-76.

[15] F. Belfiori, W. van Rossum, and P. Hoogeboom, "Application of 2d music algorithm to range-azimuth fmcw radar data," in 2012 9th European Radar Conference. IEEE, 2012, pp. 242-245.

[16] D. Feng, C. Haase-Schütz, L. Rosenbaum, H. Hertlein, C. Glaeser, F. Timm, W. Wiesbeck, and K. Dietmayer, "Deep multi-modal object detection and semantic segmentation for autonomous driving: Datasets, methods, and challenges," IEEE Transactions on Intelligent Transportation Systems, 2020.

[17] S. Gishkori and B. Mulgrew, "Azimuth enhancement for automotive sar imaging," in 2018 International Conference on Radar (RADAR). IEEE, 2018 , pp. 1-5.

[18] S. Gishkori, L. Daniel, M. Gashinova, and B. Mulgrew, "Imaging for a forward scanning automotive synthetic aperture radar," IEEE Transactions on Aerospace and Electronic Systems, vol. 55, no. 3, pp. 1420-1434, 2018.

[19] T. Kobayashi, H. Yamada, Y. Yamaguchi, and Y. Sugiyama, "Simple squint angle compensation for sar with millimeter wave automotive radar," in 2017 International Symposium on Antennas and Propagation (ISAP). IEEE, 2017, pp. 1-2.
[20] H. Yamada, T. Kobayashi, Y. Yamaguchi, and Y. Sugiyama, "Highresolution $2 \mathrm{~d}$ sar imaging by the millimeter-wave automobile radar," in 2017 IEEE Conference on Antenna Measurements \& Applications (CAMA). IEEE, 2017, pp. 149-150.

[21] R. Wang, O. Loffeld, H. Nies, S. Knedlik, M. Hagelen, and H. Essen, "Focus fmcw sar data using the wavenumber domain algorithm," IEEE Transactions on Geoscience and Remote Sensing, vol. 48, no. 4, pp. 2109-2118, 2009.

[22] E. Casalini, M. Frioud, D. Small, and D. Henke, "Refocusing fmcw sar moving target data in the wavenumber domain," IEEE Transactions on Geoscience and Remote Sensing, vol. 57, no. 6, pp. 3436-3449, 2019.

[23] A. Meta, P. Hoogeboom, and L. P. Ligthart, "Signal processing for fmcw sar," IEEE Transactions on Geoscience and Remote Sensing, vol. 45, no. 11, pp. 3519-3532, 2007.

[24] T. Kobayashi, H. Yamada, Y. Sugiyama, S. Muramatsu, and Y. Yamagchi, "Study on imaging method and doppler effect for millimeter wave automotive sar," in 2018 International Symposium on Antennas and Propagation (ISAP). IEEE, 2018, pp. 1-2.

[25] A. Ribalta, "Time-domain reconstruction algorithms for fmcw-sar," IEEE Geoscience and Remote Sensing Letters, vol. 8, no. 3, pp. 396400, 2010.

[26] E. C. Zaugg and D. G. Long, "Generalized frequency scaling and backprojection for lfm-cw sar processing," IEEE Transactions on Geoscience and Remote Sensing, vol. 53, no. 7, pp. 3600-3614, 2015.

[27] Wikipedia contributors, "Braking distance — Wikipedia, the free encyclopedia," https://en.wikipedia.org/wiki/Braking_distance, 2020, [Online; accessed 2-August-2020]. 